\title{
Association between faecal load of lawsonia intracellularis and pathological findings of proliferative enteropathy in pigs with diarrhoea
}

Ken Steen Pedersen ${ }^{1 *}$, Marie Ståhl ${ }^{2}$, Roberto Maurício Carvalho Guedes ${ }^{3}, \varnothing_{y s t e i n}$ Angen $^{2}$, Jens Peter Nielsen ${ }^{1}$ and Tim K Jensen ${ }^{2}$

\begin{abstract}
Background: The study was designed to investigate correlation between histological findings of Lawsonia intracellularis in porcine cases of diarrhoea and the quantitative detection of Lawsonia intracellularis in faeces. A total of 156 pigs (10 to 70 days post weaning) with diarrhoea were randomly selected from 20 herds: The pigs were subjected to necropsy, histopathology, immunohistochemistry and faecal quantification of Lawsonia intracellularis by real time PCR.

Results: The median Lawsonia intracellularis excretion was significantly higher in pigs with gross lesions of proliferative enteropathy (median excretion: $5.92 \log _{10}$ bacteria/g faeces) compared to pigs without gross lesions of proliferative enteropathy (median excretion: $<3.3 \log _{10}$ bacteria/g faeces) $(P<0.001)$. Spearman's correlation coefficient between the measureable PE lesions and $L$. intracellularis excretion was $0.50(P<0.001)$. A significantly increasing trend in Lawsonia intracellularis excretion level for increasing proliferative enteropathy histopathology and immunohistochemistry scores was demonstrated $(P<0.001 ; P<0.001)$. Spearman's correlation coefficient between the histopathology scores and $L$. intracellularis excretion was $0.67(P<0.001)$. Spearman's correlation coefficient between the IHC scores and $L$. intracellularis excretion was $0.77(P<0.001)$.

Conclusions: The histological and quantitative PCR detection of Lawsonia intracellularis were correlated in pigs with diarrhoea. Overall the results suggest that clinically important levels for Lawsonia intracellularis excretion in faeces may be established. Such clinical threshold levels may be used in practice to confirm a diagnosis of Lawsonia intracellularis associated diarrhoea.
\end{abstract}

Keywords: Diarrhoea, Immunohistochemistry, Lawsonia intracellularis, Quantitative real time PCR, Pig

\section{Background}

The antibiotic consumption in the agricultural industry has increasing concern in relation to development of antimicrobial resistance in both animals and humans [1]. In Denmark approximately $50 \%$ of the antibiotics in pigs is used for treatment of diarrhoea [2]. Optimizing antibiotic treatments or reducing disease occurrence necessitates a correct diagnosis. A number of intestinal pathogens have been associated with diarrhoea in growing pigs, including Escherichia coli, Brachyspira pilosicoli,

\footnotetext{
* Correspondence: ken@life.ku.dk

'HERD - Centre for Herd oriented Education, Research and Development, Department of Large Animal Sciences, University of Copenhagen, Groennegaardsvej 2, Frederiksberg C 1870, Denmark

Full list of author information is available at the end of the article
}

Brachyspira hyodysenteriae, Salmonella spp. and Lawsonia intracellularis. L. intracellularis is the cause of proliferative enteropathy (PE) and considered one of the most important intestinal pathogens [3]. Clinical signs associated with the different intestinal pathogens are similar and microbiological investigations are necessary to confirm the specific microbiological cause in cases of diarrhoea. For L. intracellularis obtaining a correct microbiological diagnosis is complicated. Subclinical infection is common [4], L. intracellularis can be demonstrated by PCR in animals vaccinated by an avirulent live vaccine [5] and the bacterial load in faeces could previously not be assessed in routine diagnostic work as the bacterium could only be cultivated and maintained in 
cell cultures [6]. Development of quantitative PCR (qPCR) tests for quantification of $L$. intracellularis in faeces [5,7-10] has now made it possible to determine the number of $L$. intracellularis bacteria in faeces on a routine basis. This may be useful for confirmation of L. intracellularis as the microbiological cause in porcine diarrhoea. Correlation between disease severity and excretion load for a specific pathogen may be expected. However, such correlation cannot always be demonstrated [11].

In this paper a quantitative real time PCR was used to investigate correlation between histological findings of L. intracellularis in porcine cases of diarrhoea and the quantity of $L$. intracellularis detected by qPCR in faeces

\section{Results}

A total of 20 outbreaks were examined (one outbreak in 20 different herds) and 160 pigs (mean days post weaning $=33$ ) were euthanized during the herd visits. Four pigs were excluded as less than 1.0 gram of faeces had been obtained, providing a total of 156 pigs with diarrhoea for the statistical analysis.

L. intracellularis was detected by $\mathrm{qPCR}$ in $25.6 \%$ of the pigs. Among the $\mathrm{qPCR}$ positive pigs $30.0 \%$ were below the dynamic range (4.3 $\log 10$ bacteria/g faeces) of the qPCR, $70.0 \%$ were in the dynamic range and no pigs were above the dynamic range ( $8.3 \log 10$ bacteria/g faeces). The median excretion for all positive pigs was $5.44 \log _{10}$ bacteria/g faeces.

\section{Gross pathology}

Gross lesions of PE were demonstrated in $8.3 \%$ of the pigs. The mean gross lesions in pigs with measureable lesions $(n=11)$ were $193 \mathrm{~cm}$ in extent (range: 17 to 600$)$. Among the pigs without gross lesions, $20.3 \%$ were qPCR positives (median excretion: $5.03 \log _{10}$ bacteria/g faeces). Among the pigs with gross lesions, $84.6 \%$ were qPCR positives (median excretion: $6.01 \log _{10}$ bacteria/g faeces). Spearman's correlation coefficient between the measureable PE lesions and L. intracellularis excretion was 0.50 $(P<0.001)$. Association between the measureable PE lesions and L. intracellularis excretion in qPCR positive pigs are displayed in Figure 1. The median L. intracellularis excretion was significantly higher in pigs with gross lesions (median excretion: $5.92 \log _{10}$ bacteria/g faeces) compared to pigs without gross lesions (median excretion: below limit of detection) $(P<0.001)$.

\section{Histopathology}

Histological lesions of PE were demonstrated in $14.1 \%$ of the pigs. Among the pigs without histological lesions, $14.9 \%$ were qPCR positives (median excretion: $4.41 \log _{10}$ bacteria/g faeces). Among the pigs with histological lesions, $90.9 \%$ were $\mathrm{qPCR}$ positives (median excretion $=$ $6.0 \log _{10}$ bacteria/g faeces.

The Kruskal-Wallis equality of populations rank tests demonstrated an overall significantly difference between the median excretion levels for the histopathology scores $(P<0.001)$. The test for trend demonstrated a significantly increasing trend in excretion level for increasing histopathology scores $(P<0.001)$. Spearman's correlation coefficient between the histopathology scores and $L$. intracellularis excretion was $0.67(P<0.001)$. Association between the histopathology score and $L$. intracellularis excretion is displayed in Figure 2.

\section{Immunohistochemistry}

L. intracellularis was detected by IHC in $19.9 \%$ of the pigs. Among the IHC negative pigs, $10.4 \%$ were qPCR positives (median excretion: below dynamic range). Among the IHC positive pigs, $87.1 \%$ were qPCR positives (median excretion $=5.83 \log _{10}$ bacteria $/ g$ faeces) . The Kruskal-Wallis equality of populations rank tests demonstrated an overall significantly difference between the median excretion levels for the IHC scores $(P<0.001)$. The test for trend demonstrated a significantly increasing trend in excretion level for increasing IHC scores $(P<0.001)$. Spearman's correlation coefficient between the IHC scores and $L$. intracellularis excretion was $0.77(P<0.001)$. Association between the IHC score and L. intracellularis excretion is displayed in Figure 3.

\section{Discussion}

Routine q-PCR quantification of a number of virus infections is now well established in medicine and has recently been reviewed [12-14]. Application of qPCR for quantification of bacterial infections is less well established in routine diagnostics, especially in veterinary medicine. However, correlation between disease severity and qPCR quantification of bacterial infections in clinical samples from animals and humans has been reported for Borrelia burgdorferi [15], Mycoplasma genitalium [16], Brucella spp. [17], Helicobacter pylori [18], Mycobacterium leprae [19,20], Mycoplasma gallisepticum [21], Streptococcus pneumoniae [22], Brucella melitensis [23], Haemophilus influenzae [24] and Legionella penumophila [25]. Further, clinical cut-off levels for bacterial load has been established for Streptococcus pneumoniae [22,26], Haemophilus influenzae [24], Mycobacterium tuberculosis [27], Gardnella vaginalis and Atopobium vaginae [28].

In the current study a positive correlation between disease severity in terms of pathological findings and quantitative detection of $L$. intracellularis in faeces was demonstrated in pigs with diarrhoea. These results are in accordance with a previous report of correlation between $L$. intracellularis bacteria load in mucosal 


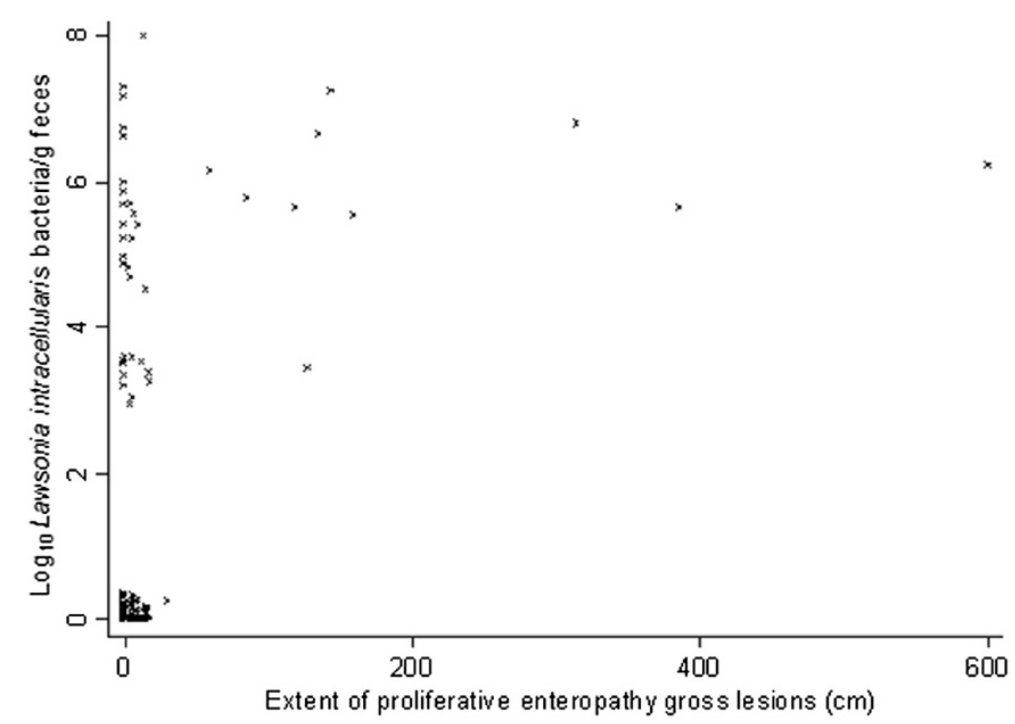

Figure 1 Association between proliferative gross lesions and Lawsonia intracellularis excretion. Association between measureable

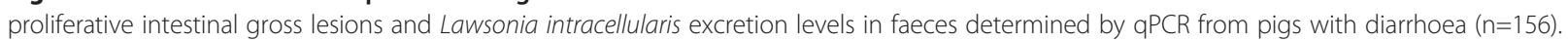

scrapings and the severity of intestinal lesions [29]. In qPCR positive pigs the median $L$. intracellularis excretion level was higher in pigs with PE gross lesions compared to pigs without gross lesions. This suggests that quantification by qPCR might be applied for examination of presence or absence of gross lesions of PE. The extent of PE gross lesions and L. intracellularis excretion levels were apparently not correlated. However, this association should be further investigated because of the low number of pigs with gross lesions in the current study.
In contrast, increasing histopathology/IHC scores were correlated to increasing $L$. intracellularis excretion levels. One interesting aspect of the observed observations is that demonstration of L. intracellularis in faeces is not evident of ileitis but in case of ileitis high excretion levels of $L$. intracellularis can be expected. The applied study design did not take progression of $L$. intracellularis infection into consideration. The obtained results are only relevant for the association between gross pathology, histopathology, IHC and quantification of L. intracellularis by $\mathrm{qPCR}$ at the time of faecal

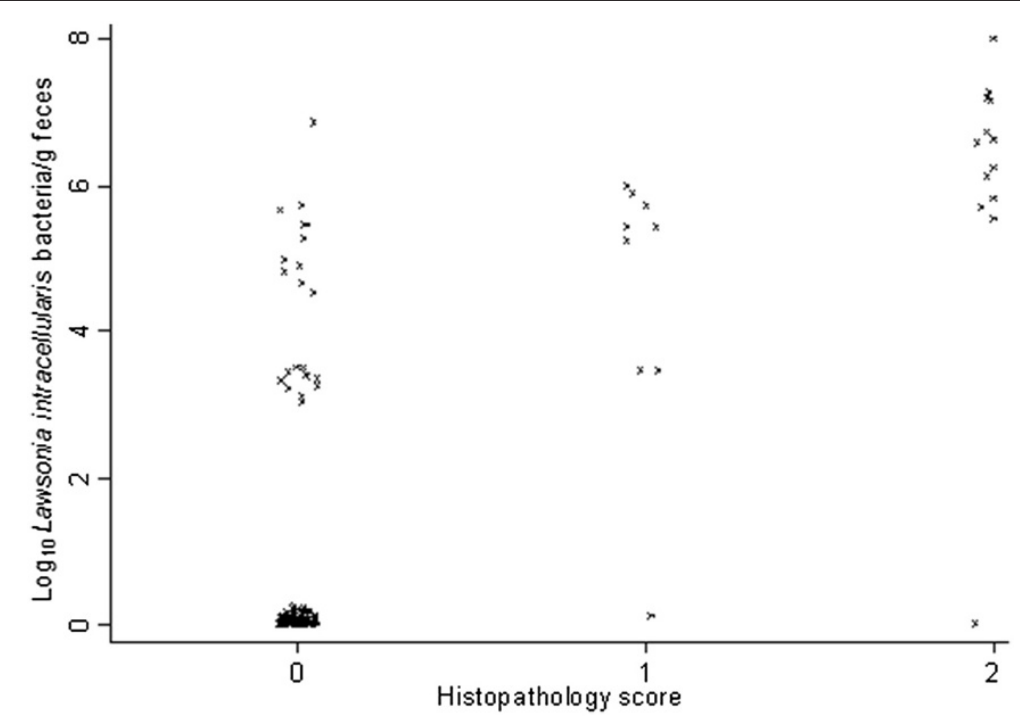

Figure 2 Association between histopathology and Lawsonia intracellularis excretion. Association between histopathology score and Lawsonia intracellularis excretion levels in faeces determined by qPCR from pigs with diarrhoea $(n=156)$. 


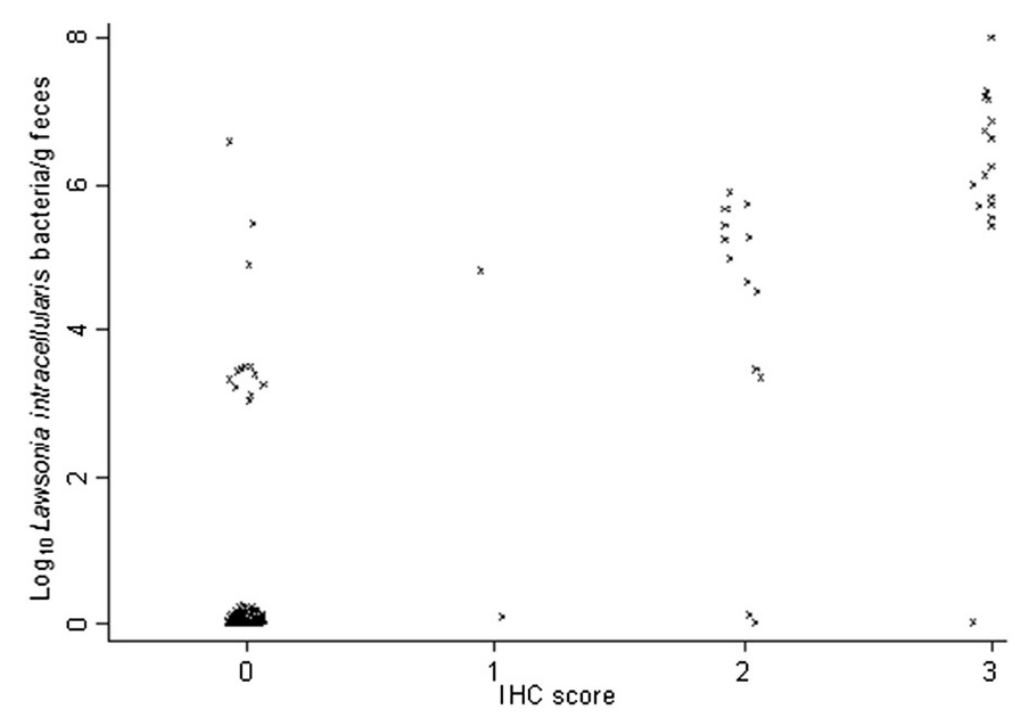

Figure 3 Association between immunohistochemistry (IHC) and Lawsonia intracellularis excretion. Association between immunohistochemistry (IHC) score and Lawsonia intracellularis excretion levels in faeces determined by qPCR from pigs with diarrhoea ( $n=156$ ).

sampling. Correlation over time and progression of L. intracellularis excretion during an infection should be performed in a longitudinal study design. Factors that potential could have influenced the results are the intermitted shedding of $L$. intracellularis previously described [30] and non-homogeneous distribution of L. intracellularis in faeces and/or intestinal tissue. Quantification of $L$. intracellularis in faeces has been reported to have an acceptable within day repeatability [31] suggesting that non-homogeneous distribution of $L$. intracellularis in faeces is not a major bias in the current study. Some of the qPCR negative animals were probably false negatives because of intermitted shedding caused by a L. intracellularis excretion level close to the qPCR's limit of detection as previously described [31]. This aspect has potentially introduced misclassification bias in the study. However, it is most likely only low excreting pigs that were classified as false negatives introducing only minor bias in the ranking of animals, the statistical analysis and the results. Inclusion of multiple standardized intestinal segments and inclusion of any gross lesions in the histological examinations have probably decreased any effect of a potential non-homogeneous distribution of L. intracellularis in the intestinal tissue.

Other factors including intestinal infections could potentially influence the reported associations. The reported lesions (IHC, proliferative lesions) are very specific for $L$. intracellularis. Therefore it seems very unlikely that other factors would have a confounding effect between these lesions and the faecal L. intracellularis excretion. However it is possible that interactions exists providing different absolute quantitative associations between $L$. intracellularis and the lesions depending on simultaneous infections or other factors. The number of observations in the current dataset did not allow for investigation of this aspect, which should be explored before clinical relevant threshold levels can be used in practice.

\section{Conclusions}

The study has demonstrated that histological and quantitative PCR detection of $L$. intracellularis is correlated in pigs with diarrhoea. Overall the results suggest that clinically important levels for L. intracellularis excretion in faeces may be established. Such clinical threshold levels may be used in practice to confirm a diagnosis of L. intracellularis associated diarrhoea, for monitoring of treatment effects or for prognostic purposes.

\section{Methods}

\section{Collection of materials from porcine diarrhoea}

No prior information concerning faecal excretion levels of $L$. intracellularis in pigs with different levels of histological findings of $L$. intracellularis was available for a formal sample size calculation. General sample size and power considerations for estimation of correlation and differences between groups for continuous outcome variables were performed using Stata IC version 11. Data previously collected for investigation of outbreaks of treatment-requiring diarrhoea in pigs $10-70$ days post weaning was considered to contain a sufficient number of pigs for the current study. In brief, farmers from 32 selected herds were requested to notify the corresponding author at initiation of an outbreak of diarrhoea in pigs between 10 and 70 days post weaning. All herds were visited the day following notification and the 
farmer was not allowed to medicate any pigs before the pigs had been examined.

To avoid any post antibiotic effect the outbreaks were not included if the pigs had received antibiotic medication in feed or water with-in the last 7 days. In each outbreak 8 pigs with diarrhoea was selected by random sampling for necropsy and collection of faecal samples. The pigs were euthanized in the herd, the abdomen was incised and intestinal specimens for histopathological examinations were collected. At the end of each herd visit the euthanized pigs and faecal samples were transported to the National Veterinary Institute, Technical University of Denmark. Pigs and faecal samples were stored at $4^{\circ}$ C. Necropsy and processing of faecal samples were performed the following day.

All parts of the study were performed in consistency to the Danish welfare legislation.

\section{Gross pathology}

The pigs were subjected to necropsy and evaluation of internal organs. Gross lesions of PE was graded as $0=$ no lesions, 1 = thickened intestinal wall and/or mucosa hyperplasia, $2=$ necrotic enteritis. Furthermore, the extension of PE gross lesions was measured.

\section{Histology}

Intestinal samples were obtained within 5 minutes of euthanasia and immediately fixated in $10 \%$ neutral buffered formalin. The intestinal tissue samples were obtained from ileum (approximately $5 \mathrm{~cm}$ from the ileo-caecal junction), one random site from jejunum and from the mid-spiral region of colon.

At necropsy further tissue samples for histology were obtained from jejunum (approximately $1.5 \mathrm{~m}$ from the ileo-caecal junction) and from any intestinal gross lesions. All samples were embedded in paraffin wax and sectioned in $3 \mu \mathrm{m}$ sections.

All segments were stained by hematoxylin and eosin (H\&E) for histopathology. Histological lesions of PE were graded on a scale from 0 to 4: Grade 0: no significant lesions; grade 1: up to $25 \%$ hyperplastic enterocytes, focal or multifocally, and reduction of the number of goblet cells; grade 2: 25 to $50 \%$ hyperplastic enterocytes, multifocally, and reduction of the number of goblet cells; grade 3: 50 to $75 \%$ hyperplastic enterocytes, multifocally, and reduction of the number of goblet cells; grade 4 : More than $75 \%$ hyperplastic enterocytes, multifocally or diffusely, and reduction of the number of goblet cells.

All segments were stained by immunohistochemistry for detection of $L$. intracellularis as previously described [32]. Results of the IHC examination were graded on a scale 0-8: Grade 0: no labelled antigen; grade 1: Up to $25 \%$ of intestinal mucosa with labelled antigen in lamina propria only; grade 2: 25 to $50 \%$ of intestinal mucosa with labelled antigen in lamina propria only; grade 3: 50 to $75 \%$ of intestinal mucosa with labelled antigen in lamina propria only; grade 4 : more than $75 \%$ of intestinal mucosa with labelled antigen in lamina propria only; grade 5: Up to $25 \%$ of intestinal mucosa with labelled antigen, enterocytes and lamina propria; grade 6: 25 to $50 \%$ of intestinal mucosa with labelled antigen, enterocytes and lamina propria; grade 7: 50 to $75 \%$ of intestinal mucosa with labelled antigen, enterocytes and lamina propria; grade 8 : more than $75 \%$ of intestinal mucosa with labelled antigen, enterocytes and lamina propria.

\section{Faecal dry matter}

All faecal samples were subjected to faecal dry matter determination to confirm the diagnosis of diarrhoea in the individual pigs. The individual faecal samples were mixed with a spoon and faecal dry matter content was determined by drying to constant weight using a microwave oven as previously described [33]. Only pigs with faecal dry matter content below 0.18 were considered diarrhoeic in the statistical analysis as previously described [33].

\section{qPCR}

A suspension of $10 \%$ faeces in phosphate buffered saline (PBS) was prepared from each faecal sample. The individual faecal samples were mixed with a plastic spoon and 0.1 gram of faeces was suspended in 0.9 gram of PBS. The faeces suspension was stored at $-80^{\circ} \mathrm{C}$ until further processing. Total DNA was extracted from the $10 \%$ faecal suspensions by using QIAsymphony extraction robot and QIAsymphony Virus/Bacteria Mini Kit (QIAGEN, GmbH, Germany). The protocol was Pathogen Complex 200, the processed sample volume was $200 \mu \mathrm{L}$ and elution was done in $110 \mu \mathrm{L}$. Prior to DNA extraction the $10 \%$ faecal suspensions were pre-treated by bead beating in Tissuelyser ( 20 sek., $15 \mathrm{~Hz}$, at room temperature, QIAGEN) with stainless steel beads ( $5 \mathrm{~mm}$, QIAGEN). The suspensions were centrifuged for 90 sek. at $10000 \mathrm{rpm}$ (MiniSpin plus, Eppendorf) and the supernatant was transferred to the QIAsymphony robot. One negative extraction sample of other bacterial cells and one positive extraction sample of $L$. intracellularis were included in each experiment. All qPCR experiments were run in duplicates as previously described [9]. The limit of detection was $3.3 \log _{10}$ bacteria/ $\mathrm{g}$ faeces and the dynamic range was $4.3-8.3 \log _{10}$ bacteria/g faeces.

\section{Statistical analysis}

Classification of individual pigs in relation to histological lesions of PE and IHC was performed before the statistical analysis. The highest grade from any intestinal segment in a pig defined the grade for that pig. To obtain 
more observations in each category, both histopathology and IHC grades were reclassified in the statistical analysis. In relation to histopathology, pigs without histopathological lesions of proliferative enteropathy were classified as score zero. Pigs with histopathology grade one or two were classified as score one and pigs with histopathology grad three or four were classified as score two. In relation to IHC, pigs observed to be negative by IHC were classified as score zero. Pigs with labeled antigen in lamina propria only (IHC grade one, two or three) were classified as score one. Pigs with IHC grad four or five were classified as score two and pigs with IHC grad six or seven were classified as score three. Overall differences in the median excretion level between pigs with different scores of gross pathology, histopathology and IHC classifications was assessed by the Kruskal-Wallis equality of populations rank test. The overall trend in excretion levels for the different scores was assessed as previously described [34] and Spearman's correlation coefficients including associated $P$ values were calculated.

\section{Competing interest}

The authors declare that they have no competing interests.

\section{Authors' contributions}

All authors conceived and designed the study. KSP performed all clinical herd investigations. RG and TKJ performed all pathological and histopathological examinations. MS and ØA performed all PCR experiments. KSP conducted the statistical analyses. All authors participated in drafting the manuscript. All authors have read and approved the final manuscript.

\section{Acknowledgements}

This work was supported by the Danish Ministry of Food, Agriculture and Fisheries. We thank the personnel at the National Veterinary Institute, Technical University of Denmark for their technical assistance.

\section{Author details}

${ }^{1}$ HERD - Centre for Herd oriented Education, Research and Development, Department of Large Animal Sciences, University of Copenhagen, Groennegaardsvej 2, Frederiksberg C 1870, Denmark. ${ }^{2}$ National Veterinary Institute, Technical University of Denmark, Bülowsvej 27, Copenhagen V 1790, Denmark. ${ }^{3}$ Department of Veterinary Clinics and Surgery, Veterinary School, Universidade Federal de Minas Gerais, Av. Antônio Carlos 6627, Belo Horizonte, MG 31.270-901, Brazil.

Received: 28 June 2012 Accepted: 15 October 2012

Published: 23 October 2012

\section{References}

1. Aarestrup FM: Association between the consumption of antimicrobial agents in animal husbandry and the occurrence of resistant bacteria among food animals. Int J Antimicrob Agents 1999, 12(4):279-285.

2. Hybschmann GK, Ersboll AK, Vigre H, Baadsgaard NP, Houe H: Herd-level risk factors for antimicrobial demanding gastrointestinal diseases in danish herds with finisher pigs: a register-based study. Prev Vet Med 2011, 98(2-3):190-197.

3. Thomson JR, Frindship RM: Digestive system. In Diseases of swine. 10th edition. Edited by Zimmerman JJ, Karriker LA, Ramirez A, Schwartz KJ, Stevenson GW. Ames: Blackwell Publishing; 2006:199-226.

4. McOrist S: Defining the full costs of endemic porcine proliferative enteropathy. Vet J 2005, 170(1):8-9.
5. Nathues $H$, Holthaus K, Beilage EG: Quantification of lawsonia intracellularis in porcine faeces by real-time PCR. J Appl Microbiol 2009, 107(6):2009-2016.

6. McOrist S, Lawson GH: Porcine proliferative enteropathy. Vet Rec 1993, 132(14):368

7. Drozd M, Rajashekara G, Kassem II, Gebreyes W: A quantitative polymerase chain reaction assay for detection and quantification of lawsonia intracellularis. J Vet Diagn Invest 2010, 22(2):265-269.

8. Richter B, Ladinig A, Nedorost N, Weissenbock H: A TaqMan quantitative polymerase chain reaction assay for the detection of lawsonia intracellularis in faecal and tissue samples from pigs. J Vet Diagn Invest 2010, 22(1):70-73.

9. Ståhl M, Kokotovic B, Hjulsager CK, Breum $S \varnothing$, Angen $\varnothing$ : The use of quantitative $P C R$ for identification and quantification of brachyspira pilosicoli, lawsonia intracellularis and escherichia coli fimbrial types F4 and F18 in pig faeces. Vet Microbiol 2011, 151(3-4):307-314.

10. Wattanaphansak S, Gebhart CJ, Anderson JM, Singer RS: Development of a polymerase chain reaction assay for quantification of lawsonia intracellularis. J Vet Diagn Invest 2010, 22(4):598-602.

11. Burns RE, Wagner DC, Leutenegger CM, Pesavento PA: Histologic and molecular correlation in shelter cats with acute upper respiratory infection. J Clin Microbiol 2011, 49(7):2454-2460.

12. Claas ECJ, Melchers WJG, van den Brule AJC: The role of real-time PCR in routine microbial diagnostics. In Real-time PCR in microbiology: from diagnosis to characterization. Edited by Mackay IM, Sakzewski A. Norfolk, UK: Caister Academic Press; 2007:245.

13. Lyon E, Wittwer CT: LightCycler technology in molecular diagnostics. J Mol Diagn 2009, 11(2):93-101.

14. Ratcliff RM, Chang G, Kok T, Sloots TP: Molecular diagnosis of medical viruses. Curr Issues Mol Biol 2007, 9(2):87-102.

15. Pahl A, Kuhlbrandt U, Brune K, Rollinghoff M, Gessner A: Quantitative detection of borrelia burgdorferi by real-time PCR. J Clin Microbiol 1999, 37(6):1958-1963.

16. Svenstrup HF, Jensen JS, Bjornelius E, Lidbrink P, Birkelund S, Christiansen G: Development of a quantitative real-time PCR assay for detection of mycoplasma genitalium. J Clin Microbio/ 2005, 43(7):3121-3128.

17. Queipo-Ortuno MI, Colmenero JD, Bravo MJ, Garcia-Ordonez MA, Morata P: Usefulness of a quantitative real-time PCR assay using serum samples to discriminate between inactive, serologically positive and active human brucellosis. Clin Microbiol Infect 2008, 14(12):1128-1134.

18. Molnar B, Szoke D, Ruzsovics A, Tulassay Z: Significantly elevated Helicobacter pylori density and different genotype distribution in erosions as compared with normal gastric biopsy specimen detected by quantitative real-time PCR. Eur J Gastroen Hepat 2008, 20(4):305-313.

19. Lini N, Shankernarayan NP, Dharmalingam K: Quantitative real-time PCR analysis of Mycobacterium leprae DNA and mRNA in human biopsy material from leprosy and reactional cases. J Med Microbiol 2009, 58(Pt 6):753-759.

20. Rudeeaneksin J, Srisungngam S, Sawanpanyalert $P$, Sittiwakin $T$ Likanonsakul S, Pasadorn S, Palittapongarnpim P, Brennan PJ, Phetsuksiri B: LightCycler real-time PCR for rapid detection and quantitation of mycobacterium leprae in skin specimens. FEMS Immunol Med Microbiol 2008, 54(2):263-270.

21. Grodio JL, Dhondt KV, O'Connell PH, Schat KA: Detection and quantification of mycoplasma gallisepticum genome load in conjunctival samples of experimentally infected house finches (carpodacus mexicanus) using real-time polymerase chain reaction. Avian Pathol 2008, 37(4):385-391.

22. Abdeldaim GM, Stralin K, Olcen P, Blomberg J, Herrmann B: Toward a quantitative DNA-based definition of pneumococcal pneumonia: a comparison of Streptococcus pneumoniae target genes, with special reference to the Spn9802 fragment. Diagn Microbiol Infect Dis 2008, 60(2):143-150

23. Castano MJ, Solera J: Chronic brucellosis and persistence of brucella melitensis DNA. J Clin Microbiol 2009, 47(7):2084-2089.

24. Abdeldaim GM, Stralin K, Kirsebom LA, Olcen P, Blomberg J, Herrmann B: Detection of haemophilus influenzae in respiratory secretions from pneumonia patients by quantitative real-time polymerase chain reaction. Diagn Microbiol Infect Dis 2009, 64(4):366-373.

25. Maurin M, Hammer L, Gestin B, Timsit JF, Rogeaux O, Delavena F, Tous J, Epaulard O, Brion JP, Croize J: Quantitative real-time PCR tests for 
diagnostic and prognostic purposes in cases of legionellosis. Clin Microbiol Infect 2010, 16(4):379-384.

26. Yang S, Lin S, Khalil A, Gaydos C, Nuemberger E, Juan G, Hardick J, Bartlett JG, Auwaerter PG, Rothman RE: Quantitative PCR assay using sputum samples for rapid diagnosis of pneumococcal pneumonia in adult emergency department patients. J Clin Microbiol 2005, 43(7):3221-3226

27. Haldar S, Sharma N, Gupta VK, Tyagi JS: Efficient diagnosis of tuberculous meningitis by detection of Mycobacterium tuberculosis DNA in cerebrospinal fluid filtrates using PCR. J Med Microbiol 2009, 58(Pt 5):616-624.

28. Menard JP, Mazouni C, Fenollar F, Raoult D, Boubli L, Bretelle F: Diagnostic accuracy of quantitative real-time $P C R$ assay versus clinical and gram stain identification of bacterial vaginosis. Eur I Clin Microbiol Infect Dis 2010, 29(12):1547-1552.

29. Guedes RMC, Gebhart CJ: Onset and duration of faecal shedding, cell-mediated and humoral immune responses in pigs after challenge with a pathogenic isolate or attenuated vaccine strain of lawsonia intracellularis. Vet Microbiol 2003, 91(2-3):135-145.

30. Smith $\mathrm{S}, \mathrm{McOrist} \mathrm{S}$ : Development of persistent intestinal infection and excretion of lawsonia intracellularis by piglets. Res Vet Sci 1997, 62:6-10.

31. Pedersen $\mathrm{KS}$, Pedersen $\mathrm{KH}$, Hjulsager $\mathrm{C}$, Larsen LE, Ståhl M, Angen Ø Stege $H$, Nielsen JP: Within-day repeatability for absolute quantification of lawsonia intracellularis bacteria in feces from growing pigs. J Vet Diagn Invest 2012, 24(5):968-970.

32. Jensen TK, Svensmark B, Vigre H, Bille-Hansen V: Distinction between porcine circovirus type 2 enteritis and porcine proliferative enteropathy caused by lawsonia intracellularis. J Comp Pathol 2006, 135(4):176-182.

33. Pedersen KS, Stege H, Nielsen JP: Evaluation of a microwave method for dry matter determination in faecal samples from weaned pigs with or without clinical diarrhoea. Prev Vet Med 2011, 100(3-4):163-170.

34. Cuzick J: A wilcoxon-type test for trend. Stat Med 1985, 4(1):87-90.

doi:10.1186/1746-6148-8-198

Cite this article as: Pedersen et al: Association between faecal load of lawsonia intracellularis and pathological findings of proliferative enteropathy in pigs with diarrhoea. BMC Veterinary Research 2012 8:198.

\section{Submit your next manuscript to BioMed Central and take full advantage of:}

- Convenient online submission

- Thorough peer review

- No space constraints or color figure charges

- Immediate publication on acceptance

- Inclusion in PubMed, CAS, Scopus and Google Scholar

- Research which is freely available for redistribution 